12 MYOCARDIAL VIABILITY IN PRESERVED OR MILDLY IMPAIRED LEFT VENTRICULAR FUNCTION PRIOR TO REVASCULARISATION - FINDINGS FROM A 3 YEAR EXPERIENCE

${ }^{1} \mathrm{H}$ Douglas, ${ }^{1} \mathrm{~B}$ Cole, ${ }^{2} \mathrm{CM}$ Soong, ${ }^{2} \mathrm{M}$ Harbinson, ${ }^{3} \mathrm{P}$ Horan, ${ }^{1} \mathrm{U}$ Dixon, ${ }^{1} \mathrm{~N}$ Johnston. ${ }^{1}$ Cardiology Department, Royal Victoria Hospital, Belfast, Northern Ireland, UK; ${ }^{2}$ Queen's University, Belfast, Northern Ireland, UK; ${ }^{3}$ Cardiology Department, Antrim Area Hospital, Antrim, Northern Ireland, UK

\subsection{6/heartjnl-2015-307845.12}

Background Viability assessment prior to revascularisation at our institution is largely requested for patients with severely impaired left ventricles (LV). This study however aims to review the outcomes of those patients with preserved or mildly impaired LV function who underwent cardiac magnetic resonance imaging (CMR) for assessment of viability prior to revascularisation and to evaluate its use in this group.

Methods All patients undergoing CMR to assess viability prior to coronary artery revascularisation were identified and included from January 2011 until June 2013.

Results Viability assessments were undertaken in 256 patients who were referred for revascularisation by either percutaneous coronary intervention (PCI) or surgery. Of these 71 (27.7\%) were found to have preserved or mildly impaired LV function, defined by an ejection fraction (EF) greater than 45\% (72\% male, mean age $66 \pm 12.8$ years). Adenosine stress perfusion was performed in $25.4 \%$. Twenty four percent of patients were turned down for revascularisation. Mean LV measurements within groups were as follows: EF CABG $56.67 \pm 7.58 \%$, PCI $57.41 \pm 8.58 \%$, medical management $59.4 \pm 19.65 \%(\mathrm{p}=0.58)$, LVEDV CABG $87.74 \pm$ $19.37 \mathrm{mls}$, PCI $85.22 \pm 20.62 \mathrm{mls}$, medical $80.41 \pm 16.14 \mathrm{mls}$ $(\mathrm{p}=0.47)$. The number of non-viable segments within each group was: CABG $2.15 \pm 1.99$, PCI $2.15 \pm 2.13$ and medical $2.41 \pm 2.06(p=0.90)$. The nonviable segments were in LAD territory in: CABG 22.2\%, PCI $33.3 \%$ and medical $29.4 \%$ $(\mathrm{p}=0.66)$. Median follow up was 2.4 years. Outcome data was follows; MACE: CABG 14.8\%, PCI 7.4\% and medical $11.8 \%$ $(\mathrm{p}=0.69)$; death: CABG $11.1 \%$, PCI $7.4 \%$, with no reported deaths in the medical management group $(\mathrm{p}=0.37)$; revascularisation: CABG 3.7\%, PCI 11.1\% and medical 11.8\% ( $\mathrm{p}=0.53)$. Conclusions Viability assessment by CMR has not been shown to have a significant impact on the management of patients with preserved or mildly impaired LV. We propose that stress perfusion for assessment of ischaemia would improve the utility of CMR in this group.

\section{CORRELATION OF INTERVENTRICULAR SYSTOLIC RELATIONSHIP AND INFARCT SIZE IN ACUTE MYOCARDIAL INFARCTION}

P Garg, A Kidambi, DP Ripley, LE Dobson, PP Swoboda, TA Musa, AK McDiarmid, B Erhayiem, JP Greenwood, S Plein. LICAMM, University of Leeds, Leeds, UK

\subsection{6/heartjnl-2015-307845.13}

Background In an echocardiographic study investigating interventricular systolic relationship in healthy individuals, the absolute value of tricuspid annular plane systolic excursion (TAPSE) is greater than the mitral annular plane of systolic excursion (MAPSE) by $54.5 \%$ and the MAPSE/TAPSE ratio is $0.66 \pm$ 0.14 . However, the interventricular systolic relationship has not been accurately defined in the presence of regional wall motion abnormalities. We hypothesised that the interventricular systolic relationship, measured by the ratio of averaged-MAPSE and
TAPSE, changes in the presence of acute myocardial infarction (AMI).

Methods Thirty-eight patients underwent CMR at 3T (Achieva CV, Philips Healthcare, Best, The Netherlands) within 3 days following AMI. Cine and LGE imaging (16-20 min following $0.1 \mathrm{mmol} / \mathrm{kg}$ gadolinium DTPA) were performed. Infarct location was determined by location of LGE in the infarcted area. MAPSE (medial, lateral and average) and TAPSE were measured from the 4-chamber cine (Figure 1). The infarct volume was measured from LGE images by Otsu method.

Results The MAPSE/TAPSE ratio in acute-MI patients was significantly reduced compared to reported normal values $(0.56 \pm$ 0.14 vs $0.66 \pm 0.14 ; \mathrm{p}=0.003)$. LV ejection fraction (EF) correlated most closely with averaged MAPSE ( $\mathrm{r}=0.45$; $\mathrm{p}=0.004)$. The interventricular systolic relationship significantly correlated with left ventricular end-diastolic indexed volume (LVEDVi) $(\mathrm{r}=-0.46 ; \mathrm{p}=0.009)$ and infarct volume $(\mathrm{r}=-0.36 ; \mathrm{p}=0.04)$.

Conclusion The ratio of averaged-MAPSE/TAPSE is significantly reduced in patients with acute-MI and correlates with infarct size. In cases where acute-MI is suspected, this parameter of interventricular systolic relationship may provide a simple additional diagnostic tool for both echocardiography and CMR assessment.

Funding JPG and SP receive a research grant from Philips Healthcare. SP is funded by British Heart Foundation fellowship (FS/10/62/28409).

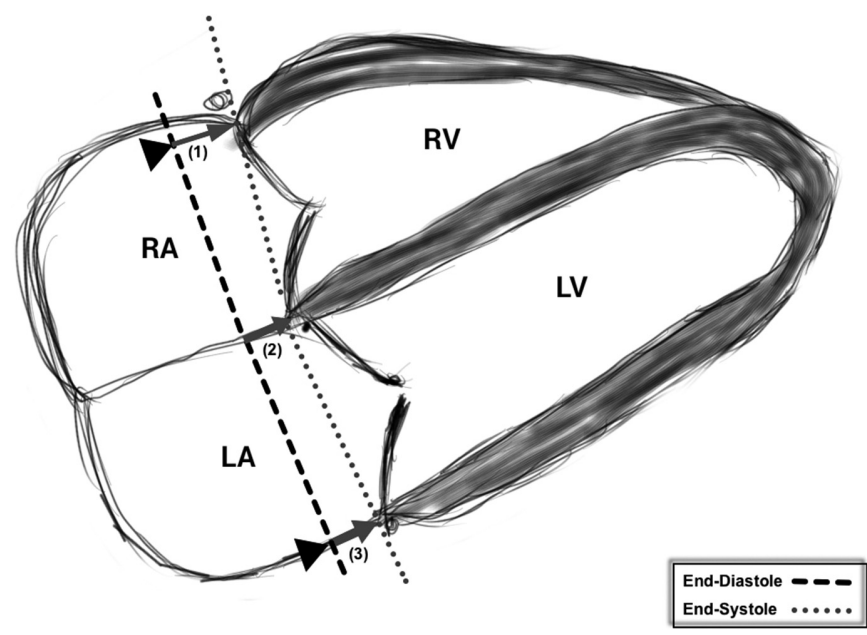

Abstract 13 Figure 1 Illustration demonstrating the three measurements. (1 - TAPSE; 2 - Medial MAPSE; 3 - Lateral MAPSE)

\section{FEATURE TRACKING VERSUS MANUAL METHODS OF ASSESSMENT OF LEFT ATRIAL MECHANICS IN ACUTE MYOCARDIAL INFARCTION: A PILOT STUDY}

P Garg, JRJ Foley, A Kidambi, DP Ripley, LE Dobson, PP Swoboda, TA Musa, AK McDiarmid, B Erhayiem, JP Greenwood, S Plein. LICAMM, University of Leeds, Leeds, UK

\subsection{6/heartjnl-2015-307845.14}

Background LA function is conventionally assessed by bi-plane method to compute LA end diastolic volume (LAEDV), end systolic volume (LAESV), stroke volume (LA SV), ejection fraction (LA EF). Voxel feature tracking (FT) is a novel technique for the assessment of LA function. We aimed to investigate if voxel FT derived longitudinal or radial strains are superior to traditionally 
derived parameters of LA function (LA SV and LA EF) in predicting LV function.

Methods Twelve-patients underwent CMR at 3T (Achieva CV, Philips Healthcare, Best, The Netherlands) within 3 days following AMI. CMR protocol included: cines and late gadolinium enhancement (LGE) imaging ( $0.1 \mathrm{mmol} / \mathrm{kg}$ gadolinium DTPA). Indexed LAEDV (LAEDVi), end-systolic volume (LAESVi) and ejection fraction were computed by bi-plane method. Voxel FT for the LA in the long axis 4-chamber cines was analysed offline using commercially available software (cvi42 v5.1, Circle Cardiovascular Imaging Inc., Calgary, Canada).

Results Demographics and basic CMR parameters are mentioned in Table 1. Analysis time was longer for manual contouring and computation of LA EF and SV compared to 4-CH strain analysis $(4 \pm 2 \mathrm{~min}$ versus $2 \pm 1.5 \mathrm{~min}, \mathrm{p}=0.01)$. On univariate analysis, LV EF was correlated to peak longitudinal strain (PLS) $(\mathrm{p}=0.01)$ and peak radial strain (PRS) $(\mathrm{p}=0.02)$. On multivariate regression analysis, PLS of LA was most strongly correlated to LV EF $(\mathrm{R}=0.68 ; \mathrm{p}=0.015)$. All other parameters did not achieve statistical significance.

Conclusion Peak left atrial longitudinal strain (PLS) is the only parameter of LA function, which independently correlates to LV ejection fraction. PLS of the LA is easily assessed on 4-chamber cines alone and takes less time to compute than the manually generated parameters of LA function.

\begin{tabular}{ll} 
Abstract 14 Table 1 & Demographics and basic CMR values \\
\hline & $\mathrm{n}=\mathbf{1 2}$ \\
\hline Age (years) & $55.4 \pm 11.2$ \\
Male & $75 \%$ \\
Hypertension & $25 \%$ \\
Hypercholesterolaemia & $42 \%$ \\
Smoker & $50 \%$ \\
LV EF & $48 \pm 10$ \\
Infarct Volume (ml) & $16.7 \pm 11.9$ \\
\hline Values expressed as $\mathrm{n}(\%)$ or mean \pm SD
\end{tabular}

\section{PREDICTORS OF RIGHT VENTRICULAR REMODELLING IN REPERFUSED INFERIOR MYOCARDIAL INFARCTIONS: CMR VOXEL FEATURE TRACKING BASED FEASIBILITY STUDY}

P Garg, A Kidambi, DP Ripley, LE Dobson, PP Swoboda, TA Musa, AK McDiarmid, B Erhayiem, P Haaf, JP Greenwood, S Plein. LICAMM, University of Leeds, Leeds, UK

\subsection{6/heartjn|-2015-307845.15}

Background RV function after STEMI has important prognostic implications. However, changes in RV function over time after inferior-STEMI and the incidence of RV remodelling remain unclear. We aimed to investigate which parameters of RV function after inferior-STEMI influence RV remodelling at 3 months. Methods Twenty-one patients underwent CMR at 3T (Achieva CV, Philips Healthcare, Best, The Netherlands) within 3-days and 90-days following reperfused inferior/posterior STEMI. The CMR protocol included: cines and LGE imaging $(0.1 \mathrm{mmol} / \mathrm{kg}$ gadolinium DTPA). Infarct location was determined from LGE images. Indexed RV end-diastolic volume (RVEDVi), end-systolic volume (RVESVi) and ejection fraction were derived from the short-axis stack cines for day-3 and day-90 scans. Offline strain analysis was performed for day-3 scans by voxel feature tracking (FT) for the RV and RA in the 4-chamber cines using commercially available software (cvi42 v5.1, Circle Cardiovascular Imaging Inc., Calgary, Canada).

Results Mean age of our population was $57 \pm 12$ years-old. $86 \%$ patients were male. RV EF improved significantly from day-3 scan to day-90 scans $(40 \pm 12.6 \%$ vs. $49 \pm 10.9 \%$, $\mathrm{p}<0.001)$. Day-3 RV EF demonstrated correlation to RV PLS $(p=0.03)$, RV PRS ( $p=0.03$ ) and RA TTP LSR (0.018). On multivariate stepwise analysis, RV PLS showed the strongest correlation $(\mathrm{R}=0.44 ; \mathrm{p}=0.04)$. Day-90 RV EF was most strongly correlated to TTP of LSR of RA $(R=0.48, p=0.048)$. Relative change in RV EF was also correlated to RV PLS $(\mathrm{p}=0.03)$.

Conclusion Voxel FT derived RV functional parameters, mainly PLS, correlates well with Day $3 \mathrm{RV}$ EF and with relative change of RV EF at day-90. Interestingly, day-90 RV EF showed the strongest correlation to time to peak longitudinal strain rate (TTP LSR) of the RA. This may be because TTP SR parameters reflect mechanical dyssynchrony after the acute ischaemic event. This concept needs further clarification in larger studies.

\section{RELATIONSHIP OF MITRAL ANNULAR PLANE SYSTOLIC EXCURSION AND INTRA-MYOCARDIAL HAEMORRHAGE IN REPERFUSED ST-ELEVATION MYOCARDIAL INFARCTION}

P Garg, A Kidambi, DP Ripley, LE Dobson, PP Swoboda, TA Musa, AK McDiarmid, B Erhayiem, JP Greenwood, S Plein. LICAMM, University of Leeds, Leeds, UK

\subsection{6/heartjnl-2015-307845.16}

Background Mitral annular plane systolic excursion (MAPSE) is known to have prognostic importance in patients with acute myocardial infarction (MI). In post-MI patients with MAPSE $<8 \mathrm{~mm}$, the combined mortality and hospitalisation incidence is $43.8 \%$. Similarly, intra-myocardial haemorrhage (IMH) in the infarct-core is an independent marker of prognosis. We hypothesised that the MAPSE on 4-chamber cine-CMR is correlated to left ventricular ejection fraction $(\mathrm{EF})$ and to the presence of IMH.

Methods Fourty-four patients received CMR examination at 3T (Achieva CV, Philips Healthcare, Best, The Netherlands) within 3 days following acute MI. Cine, T2-weighted, T2*-imaging and late gadolinium enhancement (LGE) imaging were performed. Infarct and microvascular obstruction (MO) extent were measured from LGE images. The presence and extent of IMH was investigated by combined analysis of $\mathrm{T} 2 \mathrm{w}$ and $\mathrm{T} 2 *$ sequences. MAPSE was computed (medial, lateral and average) using the 4-chamber cine (Figure 1).

Results Mean age of our studied population was 58.27 \pm 11.41. CMR parameters were as follows: LVEF $48.2 \pm 11.4 \%$; infarct volume of $15.5 \pm 12.2 \mathrm{ml}$ and averaged MAPSE of $10.27 \pm 2.1 \mathrm{~mm}$. Controlling for risk factors, IMH was strongly negatively correlated to average MAPSE $(\mathrm{r}=-0.65$; $\mathrm{p}<0.001)$. Averaged MAPSE was also moderately correlated to LVEF $(\mathrm{r}=0.47 ; \mathrm{p}=0.001)$.

Conclusion Averaged MAPSE, which is a simple CMR derived parameter of longitudinal function, has the potential to predict the presence of IMH in the setting of re-perfused acute MI. This parameter could be easily measured at bedside by transthoracic echocardiography to predict presence of IMH.

Funding JPG and SP receive a research grant from Philips Healthcare. SP is funded by British Heart Foundation fellowship (FS/10/62/28409). 